\title{
Existence of Exponential p-Stability Nonconstant Equilibrium of Markovian Jumping Nonlinear Diffusion Equations via Ekeland Variational Principle
}

\author{
Ruofeng Rao ${ }^{1,2}$ and Shouming Zhong ${ }^{3}$ \\ ${ }^{1}$ Department of Mathematics, Chengdu Normal University, Chengdu, Sichuan 611130, China \\ ${ }^{2}$ Institution of Mathematics, Yibin University, Yibin, Sichuan 644007, China \\ ${ }^{3}$ School of Mathematical Sciences, University of Electronic Science and Technology of China, Chengdu, Sichuan 611731, China \\ Correspondence should be addressed to Ruofeng Rao; ruofengrao@163.com
}

Received 18 February 2015; Accepted 14 June 2015

Academic Editor: Klaus Kirsten

Copyright (C) 2015 R. Rao and S. Zhong. This is an open access article distributed under the Creative Commons Attribution License, which permits unrestricted use, distribution, and reproduction in any medium, provided the original work is properly cited.

\begin{abstract}
The authors obtained a delay-dependent exponential $p$-stability criterion for a class of Markovian jumping nonlinear diffusion equations by employing the Lyapunov stability theory and some variational methods. As far as we know, it is the first time to apply Ekeland variational principle to obtain the existence of exponential stability equilibrium of $p$-Laplacian dynamic system so that some methods used in this paper are different from those methods of many previous related literatures. In addition, the obtained existence criterion is only involved in the activation functions so that the criterion is simpler and easier than other existence criteria to be verified in practical application. Moreover, a numerical example shows the effectiveness of the proposed methods owing to the large allowable variation range of time-delay.
\end{abstract}

\section{Introduction}

Nonlinear diffusion equations have been investigated extensively by many authors owing to their physics and biological engineering backgrounds, population dynamics, and so on (see [1-9] and references therein). In addition, Markovian jumping systems have attracted rapidly growing interest due to the fact that Markovian jumping parameters are useful in modeling abrupt phenomena, such as random failures, operating in different points of a nonlinear plant, and changing in the interconnections of subsystems (see $[8,10-12]$ and references therein). On the other hand, fuzzy logic theory has shown to be an appealing and efficient approach to deal with the analysis and synthesis problems for complex nonlinear systems. Among various kinds of fuzzy methods, Takagi-Sugeno (T-S) fuzzy models provide a successful method to describe certain complex nonlinear system using some local linear subsystems (see [13-15] and references therein). As pointed out in the above related literature, the Markovian jumping T-S fuzzy mathematical models have always found their extensive applications in the real world. However, almost all the applications are greatly dependent on the stability of systems, which can often come down to the stability of the equilibrium solution for the corresponding mathematical models. So in this paper, we may consider the stability of the nonlinear $p$-Laplace $(p>1)$ diffusion fuzzy equations with Markovian jumping. Note that when $p=2$, the so-called reaction-diffusion equations have been widely investigated (see [16-20] and references therein). For example, under Dirichlet boundary conditions, the existence result of $\mu$-stability equilibrium solution in the sense of $L^{2}$ norm for a class of time-delay reaction-diffusion equations was obtained in [16]. Motivated by the abovementioned literature, in this paper, we will synthetically employ Ekeland variational principle, Sobolev imbedding theorem, and the Lyapunov functional method to study the existence of exponential $p$-stability nonconstant equilibrium solution in the sense of $L^{p}$ norm $(p>1)$ for delayed Markovian jumping fuzzy equations with nonlinear $p$-Laplace diffusion $(p>1)$.

Let $\{r(t):[0,+\infty) \rightarrow S\}$ be a right-continuous Markov process on the complete probability space $(\mathfrak{S}, F, \mathbb{P})$ with 
a natural filtration $\left\{F_{t}\right\}_{t \geqslant 0}$ and take values in the finite space $S \triangleq\{1,2, \ldots, N\}$ with generator $\Pi=\left\{\pi_{i k}\right\}$ given by

$$
\begin{aligned}
\mathbb{P}(r(t+\delta)=k \mid r(t)=i) & = \begin{cases}\pi_{i k} \delta+o(\delta), & k \neq i, \\
1+\pi_{i k} \delta+o(\delta), & k=i,\end{cases}
\end{aligned}
$$

where $\pi_{i k} \geqslant 0$ is transition probability rate from $i$ to $k(k \neq i)$ and $\pi_{i i}=-\sum_{k \in S, k \neq i} \pi_{i k}, \quad \delta>0$, and $\lim _{\delta \rightarrow 0}(o(\delta) / \delta)=0$.

Consider the following fuzzy T-S Markovian jumping $p$ Laplace partial dynamic equations.

Fuzzy rule $j$ : IF $\omega_{1}$ is $v_{j 1}$ and ... $\omega_{\kappa}$ is $v_{j \kappa}$ THEN

$$
\begin{aligned}
\frac{d u_{i}(t, x)}{d t}= & \mathscr{D}_{i} \operatorname{div}\left(\left|\nabla u_{i}\right|^{p-2} \nabla u_{i}\right)-b_{i}\left(u_{i}\right) \\
& +c_{i j}(r(t)) f_{i}\left(u_{i}(t, x)\right) \\
& +d_{i j}(r(t)) g_{i}\left(u_{i}\left(t-\tau_{i}(t), x\right)\right)+I_{i}, \\
& \quad i \in \mathcal{N}, t>0, x \in \Omega,
\end{aligned}
$$

$$
\begin{aligned}
& u_{i}(t, x)=0, \quad i \in \mathcal{N}, t \geqslant 0, x \in \partial \Omega, \\
& u_{i}(s, x)=\phi_{i}(s, x), \\
& -\tau_{i}(0) \leqslant s \leqslant 0,0 \leqslant \tau_{i}(t) \leqslant \tau_{i}, i \in \mathcal{N}, t \geqslant 0, x \in \Omega,
\end{aligned}
$$

where $\omega_{k}(t)(k=1,2, \ldots, \kappa)$ is the premise variable and $v_{j k}(j=1,2, \ldots, J ; k=1,2, \ldots, \kappa)$ is the fuzzy set that is characterized by membership function. $J$ is the number of the IF-THEN rules and $\kappa$ is the number of the premise variables. Denote the premise variable vector $\omega(t)=\left[\omega_{1}(t), \omega_{2}(t), \ldots, \omega_{\kappa}(t)\right]$ and $\rho_{j}(\omega(t))=$ $w_{j}(\omega(t)) / \sum_{k=1}^{J} w_{k}(\omega(t))$, where $w_{j}(\omega(t)): R^{\kappa} \rightarrow[0,1](j=$ $1,2, \ldots, J)$ is the membership function of the system with respect to the fuzzy rule $j . \rho_{j}$ can be regarded as the normalized weight of each IF-THEN rule, satisfying

$$
\rho_{j}(\omega(t)) \geqslant 0, \quad \sum_{j=1}^{J} \rho_{j}(\omega(t))=1 .
$$

Using a singleton fuzzifier, product fuzzy inference, and weighted average defuzzifier, system (2) is inferred as follows:

$$
\begin{aligned}
& \frac{d u_{i}(t, x)}{d t}=\mathscr{D}_{i} \operatorname{div}\left(\left|\nabla u_{i}\right|^{p-2} \nabla u_{i}\right)-b_{i}\left(u_{i}\right) \\
& \quad+\sum_{j=1}^{J} \rho_{j}(\omega(t))\left(c_{i j}(r(t)) f_{i}\left(u_{i}(t, x)\right)\right. \\
& \left.\quad+d_{i j}(r(t)) g_{i}\left(u_{i}\left(t-\tau_{i}(t), x\right)\right)\right)+I_{i}, \\
& \quad i \in \mathcal{N}, t>0, x \in \Omega, \\
& u_{i}(t, x)=0, \quad i \in \mathcal{N}, t \geqslant 0, x \in \partial \Omega
\end{aligned}
$$

with the initial condition

$$
\begin{aligned}
& u_{i}(s, x)=\phi_{i}(s, x), \\
& -\tau_{i}(0) \leqslant s \leqslant 0,0 \leqslant \tau_{i}(t) \leqslant \tau_{i}, i \in \mathcal{N}, t \geqslant 0, x \in \Omega,
\end{aligned}
$$

where $\Omega$ is a bounded subset in $R^{m}$ with smooth boundary $\partial \Omega . p>1$ is a scalar, and $\mathcal{N}=\{1,2, \ldots, n\}$ is a finite set. The divergence $\operatorname{div}\left(\left|\nabla u_{i}(t, x)\right|^{p-2} \nabla u_{i}(t, x)\right)=\Delta_{p} u_{i}(t, x)$ is the $p$ Laplacian (see [21] for details). In mode $r(t)=r$, we denote $c_{i j}(r(t))=c_{i j}^{r}$ and $d_{i j}(r(t))=d_{i j}^{r}$, which imply the connection strengths of the $i$ th neuron on the $j$ th neuron at the system mode $r(t)=r$, respectively. For any given $i \in \mathcal{N}, \mathscr{D}_{i}>0$ is a corresponding constant, dependent on $i$.

Remark 1. Our methods employed in this paper are different from those of previous related literature. For example, homomorphic mapping theory was employed to obtain the existence of equilibrium of ordinary differential equations in [22]; topological degree theory was used to obtain the existence of equilibrium for fuzzy ordinary differential equations in [23] and of equilibrium for reaction-diffusion partial differential equations in [17]. In this paper, Ekeland variational principle is originally proposed to solve the existence of nonconstant equilibrium for nonlinear diffusion equations. Note that the abovementioned constant equilibrium point $u^{*}=\left(u_{1}^{*}, \ldots, u_{n}^{*}\right)^{T}$ can actually be regarded as the special case of our nonconstant equilibrium point $u=u^{*}(x)=$ $\left(u_{1}^{*}(x), \ldots, u_{n}^{*}(x)\right)^{T}$ with $u_{i}^{*}(x) \equiv$ constant for $i=1,2, \ldots, n$. In addition, our criterion about existence is only involved in the activation functions while other more parameters need be considered in the proof of the existence of constant equilibrium point in those previous literatures (see Remark 8 below for details).

\section{Preparation}

Throughout this paper, we assume the following:

(A1) There exists a positive definition matrix $B=\operatorname{diag}\left(B_{1}\right.$, $\left.B_{2}, \ldots, B_{n}\right)$ such that

$$
\inf _{s \in R} b_{i}^{\prime}(s) \geqslant B_{i}>0, \quad \forall i \in \mathcal{N} .
$$

(A2) There exist positive definite matrices $F=\operatorname{diag}\left(F_{1}, F_{2}\right.$, $\left.\ldots, F_{n}\right)$ and $G=\operatorname{diag}\left(G_{1}, G_{2}, \ldots, G_{n}\right)$ such that

$$
\begin{aligned}
& \left|f_{i}(s)-f_{i}(t)\right| \leqslant F_{i}|s-t|, \\
& \left|g_{i}(s)-g_{i}(t)\right| \leqslant G_{i}|s-t|,
\end{aligned}
$$

$$
s, t \in R, i \in \mathcal{N} \text {. }
$$

Definition 2. $u=u^{*}(x)=\left(u_{1}^{*}(x), u_{2}^{*}(x), \ldots, u_{n}^{*}(x)\right)^{T}$ is called a nonconstant equilibrium solution of PDEs (4) if and only if $u=u^{*}(x)$ satisfies (4). In addition, the nonconstant equilibrium solution $u^{*}(x)$ of PDEs (4) is called stochastically global exponential $p$-stability about $L^{p}$ norm, if there are constants $M>0$ and $\delta>0$ for every stochastic field solution $u(t, x)=\left(u_{1}(t, x), u_{2}(t, x), \ldots, u_{n}(t, x)\right)^{T}$ of PDEs (4) such that

$$
E\left(\left\|u(t, x)-u^{*}\right\|_{L^{p}}\right) \leqslant M e^{-\delta\left(t-t_{0}\right)},
$$

where one denotes by $\|u\|_{L^{p}}=\left(\sum_{i=1}^{n}\left\|u_{i}\right\|_{L^{p}}^{p}\right)^{1 / p}$ the $L^{p}$ norm and denotes $\left\|u_{i}\right\|_{L^{p}}=\left(\int_{\Omega}\left|u_{i}\right| d x\right)^{1 / p}$. Usually, $t_{0}=0$. 
Definition 3. Let $X$ be a Banach space, $\varphi \in C^{1}(X, R)$, and $c \epsilon$ $R$. $\varphi$ satisfies the $(\mathrm{PS})_{c}$ condition if any sequence $\left\{u_{n}\right\} \subset X$, such that

$$
\begin{gathered}
\varphi\left(u_{n}\right) \longrightarrow c, \\
\varphi^{\prime}\left(u_{n}\right) \longrightarrow 0,
\end{gathered}
$$

has a convergent subsequence. By the way, the above sequence $\left\{u_{n}\right\}$ with $\varphi\left(u_{n}\right) \rightarrow c$ and $\varphi^{\prime}\left(u_{n}\right) \rightarrow 0$ is called the $(\mathrm{PS})_{c}$ sequence of $\varphi$ for a given $c \in R$.

The following lemma originated from the famous Sobolev imbedding theorem.

Lemma 4. Let $\Omega$ be a bounded subset in $R^{m}$ with smooth boundary $\partial \Omega$. For $1<q<p$, there exist the corresponding positive constants $c_{1}$ and $c_{q}$ such that, for any $\xi \in W_{0}^{1, p}(\Omega)$,

$$
\begin{array}{r}
\int_{\Omega}|\xi| d x \leqslant c_{1}\|\xi\|, \\
\left(\int_{\Omega}|\xi|^{q} d x\right)^{1 / q} \leqslant c_{q}\|\xi\|,
\end{array}
$$

where the Sobolev space $W_{0}^{1, p}(\Omega)$ is the completion of $C_{0}^{\infty}(\Omega)$ with respect to the norm $\|\xi\|=\left(\int_{\Omega}|\nabla \xi|^{p} d x\right)^{1 / p}$.

In 1979, Ekeland proposed the following famous Ekeland variational principle and its proof in [24]. As is well known, Ekeland variational principle has been the most important result in nonlinear analysis and has been applied to optimization theory, control theory, economic equilibrium theory, critical point theory, dynamic systems, and so forth. In this paper, we also need the following Ekeland variational principle.

Lemma 5 (Ekeland variational principle [24, Theorem 1]). Let $X$ be a complete metric space and let $\varphi: X \rightarrow(-\infty,+\infty]$ be a lower semicontinuous function, bounded from below and not identical to $+\infty$. Let $\varepsilon>0$ be given and let $\xi \in X$ be such that

$$
\varphi(\xi) \leqslant \inf _{X} \varphi+\varepsilon
$$

Then there exists $v \in X$ such that

$$
\begin{aligned}
\varphi(v) & \leqslant \varphi(\xi), \\
\operatorname{dist}(\xi, v) & \leqslant 1,
\end{aligned}
$$

and, for each $w \neq v$ in $X$,

$$
\varphi(w)>\varphi(v)-\varepsilon \operatorname{dist}(v, w) .
$$

\section{Main Result}

Theorem 6. Let $p>1$. Assume that there exists a positive scalar $q=q_{1} / q_{2}$ with $1<q<p$ such that

$$
\begin{aligned}
\lim _{|s| \rightarrow+\infty} \frac{b_{i}(s)}{s^{q-1}} & =\lim _{|s| \rightarrow+\infty} \frac{f_{i}(s)}{s^{q-1}}=\lim _{|s| \rightarrow+\infty} \frac{g_{i}(s)}{s^{q-1}}=0 \\
& =b_{i}(0), \quad \forall i \in \mathcal{N},
\end{aligned}
$$

where $q_{1}$ is an odd number and so is $q_{2}$. Assume, in addition, $\left|I_{i}\right|<M$.

If there exist a sequence of positive scalars $h_{i}(r(t))(r \in$ $S, i \in \mathcal{N}$ ) such that

$$
-\mathfrak{A}+\frac{e^{\beta \tau}}{1-K} \mathfrak{B}<0,
$$

where $\tau=\max _{i \in \mathcal{N}} \tau_{i}, \tau_{i}^{\prime}(t) \leqslant K<1$ for all $i \in \mathcal{N}, \mathfrak{A}=$ $\operatorname{diag}\left(a_{1}, a_{2}, \ldots, a_{n}\right), \mathfrak{B}=\operatorname{diag}\left(b_{1}, b_{2}, \ldots, b_{n}\right), a_{i}=\min _{r \in S} a_{i}(r)$, $b_{i}=\max _{r \in S} b_{i}(r)$, and

$$
\begin{aligned}
& a_{i}(r)=h_{i}(r(t))\left[p B_{i}-\beta-p F_{i}\left(\sum_{j=1}^{J}\left|c_{i j}^{r}\right|\right)\right. \\
& \left.\quad-(p-1) G_{i}\left(\sum_{j=1}^{J}\left|d_{i j}^{r}\right|\right)\right], \\
& b_{i}(r)=h_{i}(r(t)) G_{i}\left(\sum_{j=1}^{J}\left|d_{i j}^{r}\right|\right),
\end{aligned}
$$

$r \in S$

then there exists a nonconstant equilibrium solution for PDEs (4), which is stochastically global exponential p-stability about $L^{p}$ norm.

Proof. The following proof may be divided into two big steps.

Step 1. Firstly, we need prove that there exists a nonconstant equilibrium solution for (4).

Consider the functional

$$
\begin{aligned}
\varphi_{i}(\eta) & =\int_{\Omega}\left[\frac{1}{p} \mathscr{D}_{i}|\nabla \eta|^{p}+\mathscr{B}_{i}(\eta)\right. \\
& \left.-\sum_{j=1}^{J} \rho_{j}(\omega(t))\left(\left(c_{i j}^{r} \mathscr{F}_{i}(\eta)+d_{i j}^{r} \mathscr{G}_{i}(\eta)\right)\right)-I_{i} \eta\right] d x,
\end{aligned}
$$

where $\mathscr{B}_{i}(s)=\int_{0}^{s} b_{i}(\theta) d \theta, \mathscr{F}_{i}(s)=\int_{0}^{s} f_{i}(\theta) d \theta, \mathscr{G}_{i}(s)=$ $\int_{0}^{s} g_{i}(\theta) d \theta$, and $\eta=\eta(t, x) \in W_{0}^{1, p}(\Omega)$, where the Sobolev space $W_{0}^{1, p}(\Omega)$ is the completion of $C_{0}^{\infty}(\Omega)$ with respect to the norm $\|\eta\|=\left(\int_{\Omega}|\nabla \eta|^{p} d x\right)^{1 / p}$.

It is obvious that $\varphi_{i} \in C^{1}\left(W_{0}^{1, p}(\Omega), R\right)$, for all $i \in$ $\mathcal{N}$. If its critical point $u_{i}^{*}(x)$ exists, then $u^{*}(x)=\left(u_{1}^{*}(x)\right.$, $\left.u_{2}^{*}(x), \ldots, u_{n}^{*}\left(x_{n}\right)\right)^{T}$ must be a nonconstant equilibrium solution of (4). So we only need to prove the existence of the critical point of $\varphi_{i}$ for all $i \in \mathcal{N}$.

Next, it follows from (13) that there exists a large enough $a_{M}>0$ such that

$$
\left|f_{i}(s)\right|+\left|g_{i}(s)\right| \leqslant|s|^{q-1}, \quad|s|>a_{M} .
$$

Furthermore, we can conclude by the continuity of $f_{i}$ and $g_{i}$ that

$$
\left|f_{i}(s)\right|+\left|g_{i}(s)\right| \leqslant b_{M}, \quad \forall|s| \leqslant a_{M}
$$


and hence,

$$
\left|f_{i}(s)\right|+\left|g_{i}(s)\right| \leqslant b_{M}+|s|^{q-1}, \quad \forall s \in R,
$$

where $b_{M}>0$ is a constant.

For $s \geqslant 0$, we can derive by $q=q_{1} / q_{2}$ and the restrictive conditions on $q_{1}, q_{2}$

$$
\left|\mathscr{F}_{i}(s)\right| \leqslant \int_{0}^{s}\left|f_{i}(y)\right| d y \leqslant b_{M} s+\frac{1}{q} s^{q}=b_{M}|s|+\frac{1}{q}|s|^{q},
$$

where $b_{M}>0$ is a constant.

On the other hand, for $s<0$, we can get by $q=q_{1} / q_{2}$ and the restrictive conditions on $q_{1}, q_{2}$

$$
\begin{aligned}
\left|\mathscr{F}_{i}(s)\right| & \leqslant \int_{s}^{0}\left|f_{i}(y)\right| d y \leqslant \int_{s}^{0}\left(b_{M}+|y|^{q-1}\right) d y \\
& \leqslant b_{M}|s|+\frac{1}{q}|s|^{q} .
\end{aligned}
$$

Hence,

$$
\left|\mathscr{F}_{i}(s)\right| \leqslant b_{M}|s|+\frac{1}{q}|s|^{q}, \quad \forall s \in R .
$$

Similarly, we can also deduce that

$$
\left|\mathscr{G}_{i}(s)\right| \leqslant b_{M}|s|+\frac{1}{q}|s|^{q}, \quad \forall s \in R
$$

Denote $\left|c_{i j}\right|=\max _{r \in S}\left|c_{i j}^{r}\right|$ and $\left|d_{i j}\right|=\max _{r \in S}\left|d_{i j}^{r}\right|$; then $\left|c_{i j}\right| \geqslant 0$ and $\left|d_{i j}\right| \geqslant 0$ are constants, independent of $r$. Similarly, we can prove that there exist $a_{i 1}>0$ and $a_{i 2}>0$ such that

$$
\begin{aligned}
& \sum_{j=1}^{J} \rho_{j}(\omega(t))\left(c_{i j}^{r} \mathscr{F}_{i}(s)+d_{i j}^{r} \mathscr{G}_{i}(s)\right) \\
& \leqslant\left(\sum_{j=1}^{J}\left|c_{i j}\right|\right)\left|\mathscr{F}_{i}(s)\right|+\left(\sum_{j=1}^{J}\left|d_{i j}\right|\right)\left|\mathscr{G}_{i}(s)\right| \\
& \leqslant a_{i 1}|s|+a_{i 2}|s|^{q}, \quad \forall s \in R,
\end{aligned}
$$

where $a_{i 1}=b_{M}\left(\sum_{j=1}^{J}\left|c_{i j}\right|+\sum_{j=1}^{J}\left|d_{i j}\right|\right)$ and $a_{i 2}=$ $(1 / q)\left(\sum_{j=1}^{J}\left|c_{i j}\right|+\sum_{j=1}^{J}\left|d_{i j}\right|\right)$ are the constants, independent of $r$.

Thereby, we have

$$
\begin{gathered}
{\left[\sum_{j=1}^{J} \rho_{j}(\omega(t))\left(c_{i j}^{r} \mathscr{F}_{i}(s)+d_{i j}^{r} \mathscr{G}_{i}(s)\right)\right]+I_{i} s} \\
\leqslant\left(a_{i 1}+M\right)|s|+a_{i 2}|s|^{q}, \quad \forall s \in R .
\end{gathered}
$$

Since $b_{i}^{\prime}(s)>0$, we know that $b_{i}(s)>b_{i}(0)=0$ if $s>0$ and $b_{i}(s)<b_{i}(0)=0$ if $s<0$. And hence $\mathscr{B}_{i}(s)=\int_{0}^{s} b_{i}(\theta) d \theta \geqslant 0$ for all $s \in R$. Besides, we know from the above analysis and the Sobolev imbedding theorem (Lemma 4) that there exist positive constants $b_{i 1}=c_{1}\left(a_{i 1}+M\right)$ and $b_{i 2}=c_{q}^{q} a_{i 2}$, independent of $r$, such that

$$
\begin{aligned}
\varphi_{i}(\eta) \geqslant & \frac{1}{p} \mathscr{D}_{i}\|\eta\|^{p}-\left(a_{i 1}+M\right) \int_{\Omega}|\eta| d x \\
& -a_{i 2} \int_{\Omega}|\eta|^{q} d x \\
\geqslant & \frac{1}{p} \mathscr{D}_{i}\|\eta\|^{p}-b_{i 2}\|\eta\|^{q}-b_{i 1}\|\eta\|, \quad i \in \mathcal{N} .
\end{aligned}
$$

Denote $\mu(s)=(1 / p) \mathscr{D}_{i} s^{p}-b_{i 2} s^{q}-b_{i 1} s$. Owing to $p>q>1$, there exists a large enough constant $s_{0}>0$ such that $\mu(s)>0$ for all $|s|>s_{0}$. And hence

$$
\mu(s) \geqslant \min \left\{0, \min _{s \in\left[-s_{0}, s_{0}\right]} \mu(s)\right\}, \quad s \in R,
$$

which implies that $\varphi_{i}$ is bounded below. And the infimum may be defined as $c_{i}=\inf _{X} \varphi_{i}$, where we denote $X=W_{0}^{1, p}(\Omega)$ for convenience and denote by $X^{*}$ its dual space. Define the operators $\mathbb{A}_{i}, \mathbb{B}_{i}: X \rightarrow X^{*}$ as follows:

$$
\begin{aligned}
& \left\langle\mathbb{A}_{i}(\eta), \xi\right\rangle=\mathscr{D}_{i} \int_{\Omega}|\nabla \eta|^{p-2} \nabla \eta \nabla \xi d x, \\
& \left\langle\mathbb{B}_{i}(\eta), \xi\right\rangle=\int_{\Omega}\left(b_{i}(\eta)\right. \\
& \left.\quad-\sum_{j=1}^{J} \rho_{j}(\omega(t))\left(c_{i j}^{r} f_{i}(\eta)+d_{i j}^{r} g_{i}(\eta)\right)-I_{i}\right) \xi d x,
\end{aligned}
$$

where $\eta, \xi \in X,\langle\cdot, \cdot\rangle$ denotes the adjoint pair for $X$ and $X^{*}$. It follows by (A1) and (A2) that all $b_{i}, f_{i}, g_{i}$ are continuous. Similarly to (19), we can get

$$
\left|b_{i}(s)\right|+\left|f_{i}(s)\right|+\left|g_{i}(s)\right| \leqslant c_{M}+|s|^{q-1}, \quad \forall s \in R,
$$

where $c_{M}$ is a constant. we can conclude from (13) and (29) that there exist positive constants $a_{i 3}$ and $a_{i 4}$, independent of $r$, such that

$$
\begin{gathered}
\left|b_{i}(s)-\sum_{j=1}^{J} \rho_{j}(\omega(t))\left(c_{i j}^{r} f_{i}(s)+d_{i j}^{r} g_{i}(s)\right)-I_{i}\right| \\
\leqslant c_{M}+|s|^{q-1}+\sum_{j=1}^{J}\left|c_{i j}\right| \cdot\left|f_{i}(s)\right|+\left(\sum_{j=1}^{J}\left|d_{i j}^{r}\right|\right) \\
\cdot\left|g_{i}(s)\right|+M \leqslant a_{i 3}+a_{i 4}|s|^{q-1}, \quad s \in R .
\end{gathered}
$$

Here, $a_{i 3}=M+c_{M}+c_{M}\left(\sum_{j=1}^{J}\left|c_{i j}\right|+\sum_{j=1}^{J}\left|d_{i j}\right|\right)$ and $a_{i 4}=$ $1+\sum_{j=1}^{J}\left|c_{i j}\right|+\sum_{j=1}^{J}\left|d_{i j}\right|$.

So we know from $[25,26]$ that both $\mathbb{A}_{i}$ and $\mathbb{A}_{i}^{-1}$ are continuous, and $\mathbb{B}_{i}$ is compact.

Next, we claim that, for each $i \in \mathcal{N}$, the functional $\varphi_{i}$ must satisfy the $(\mathrm{PS})_{c}$ condition if only every $(\mathrm{PS})_{c}$ sequence of $\varphi_{i}$ is bounded. 
Indeed, let $\left\{\eta_{n}\right\}$ be the $(\mathrm{PS})_{c}$ sequence of $\varphi_{i}$ for any given c. It is obvious that

$$
\left\langle\varphi_{i}^{\prime}\left(\eta_{n}\right), \xi\right\rangle=\left\langle\mathbb{A}_{i}\left(\eta_{n}\right)-\mathbb{B}_{i}\left(\eta_{n}\right), \xi\right\rangle, \quad \forall \xi \in X .
$$

Owing to $\varphi_{i}^{\prime}\left(\eta_{n}\right) \rightarrow 0$, we have $\left(\mathbb{A}_{i}\left(\eta_{n}\right)-\mathbb{B}_{i}\left(\eta_{n}\right)\right) \rightarrow$ 0 . If $\left\{\eta_{n}\right\}$ is bounded, we know from the reflexivity of the Banach space $W_{0}^{1, p}(\Omega)$ that there exists a weakly convergent subsequence (say, $\left\{\eta_{n}\right\}$ ). Since $\mathbb{B}_{i}$ is compact, $\left\{\mathbb{B}_{i}\left(\eta_{n}\right)\right\}$ must own a convergent subsequence, which implies that $\mathbb{A}_{i}\left(\eta_{n}\right)$ must own a convergent subsequence. And furthermore, the continuity of $\mathbb{A}_{i}^{-1}$ yields that $\left\{\eta_{n}\right\}$ owns a convergent subsequence.

Below, we only need to prove that every $(\mathrm{PS})_{\mathcal{c}_{i}}$ sequence (say, $\left\{\eta_{n}\right\}$ ) of $\varphi_{i}$ is bounded.

Indeed, similarly to (26), we have

$$
\varphi_{i}\left(\eta_{n}\right) \geqslant \frac{1}{p} \mathscr{D}_{i}\left\|\eta_{n}\right\|^{p}-b_{i 2}\left\|\eta_{n}\right\|^{q}-b_{i 1}\left\|\eta_{n}\right\|
$$

Owing to the boundedness of $\left\{\varphi_{i}\left(\eta_{n}\right)\right\}$, it is not difficult to prove by the application of reduction to absurdity that $\left\{\eta_{n}\right\}$ must be bounded in $W_{0}^{1, p}(\Omega)$.

Now we may define the metric for the space $X$ as follows:

$$
\operatorname{dist}(\eta, \xi)=\|\eta-\xi\| \text {. }
$$

Then $X$ is a complete metric space with the above metric. From the continuity of $\varphi_{i}$ and the above analysis, we know that $\varphi: W_{0}^{1, p}(\Omega) \rightarrow(-\infty,+\infty]$ is a lower semicontinuous function and bounded from below. Owing to $0 \in W_{0}^{1, p}(\Omega)$, we can compute and deduce that $\left|\varphi_{i}(0)\right| \leqslant \sum_{j=1}^{J}\left(\left|c_{i j}\right|\left|\mathscr{F}_{i}(0)\right|+\right.$ $\left.\left|d_{i j}\right|\left|\mathscr{G}_{i}(0)\right|\right)$, which implies $\varphi_{i} \not \equiv+\infty, \forall i \in \mathscr{N}$.

According to Ekeland variational principle, for given $\varepsilon=$ $1 / n$, there exists $\eta_{n} \in X$ such that

$$
\begin{aligned}
\varphi_{i}(\eta) & >\varphi_{i}\left(\eta_{n}\right)-\frac{1}{n}\left\|\eta-\eta_{n}\right\|, \quad \forall \eta \neq \eta_{n}, \\
\varphi_{i}\left(\eta_{n}\right) & <c_{i}+\frac{1}{n} .
\end{aligned}
$$

So we can deduce from (34)

$$
\left\|\varphi_{i}^{\prime}\left(\eta_{n}\right)\right\|=\sup _{\|\xi\|=1}\left|d \varphi_{i}\left(\eta_{n}, \xi\right)\right| \leqslant \frac{1}{n} .
$$

Here, $d \varphi_{i}\left(\eta_{n}, \xi\right)$ is Gâteaux derivative of $\varphi_{i}$ at $\eta_{n}$, and $\varphi_{i}^{\prime}\left(\eta_{n}\right)$ is Fréchet derivative of $\varphi_{i}$ at $\eta_{n}$. Besides, (35) yields $\varphi_{i}\left(\eta_{n}\right) \rightarrow c_{i}$. Then we can conclude from the $\mathrm{PS}_{c_{i}}$ condition that there exist a convergent subsequence $\left\{\eta_{n_{j}}\right\} \subset\left\{\eta_{n}\right\}$ and $u_{i}^{*} \in W_{0}^{1, p}(\Omega)$ such that $\eta_{n_{j}} \rightarrow u_{i}^{*}$. Moreover, the continuity of $\varphi_{i}$ yields $\varphi_{i}\left(u_{i}^{*}\right)=c_{i}$. Hence, $\varphi_{i}$ has a critical point $u_{i}^{*}(x)$ in $X$ for all $i \in$ $\mathcal{N}$. That is, there exists an equilibrium $u^{*}=\left(u_{1}^{*}, u_{2}^{*}, \ldots, u_{n}^{*}\right)^{T}$ for (4).

Step 2. Below, we will prove the exponential $p$-stability for the equilibrium point $u^{*}$.
Consider the Lyapunov-Krasovskii functional as

$$
V_{i}(t, r(t))=V_{1 i}(t, r(t))+V_{2 i}(t, r(t)) \quad i \in \mathcal{N},
$$

where

$$
\begin{aligned}
& V_{1 i}(t, r(t))=e^{\beta t} \int_{\Omega} h_{i}(r(t))\left|u_{i}(t, x)-u_{i}^{*}(x)\right|^{p} d x, \\
& V_{2 i}(t, r(t)) \\
& \quad=\frac{b_{i}}{1-K} e^{\beta \tau} \int_{\Omega} \int_{t-\tau_{i}(t)}^{t} e^{\beta s}\left|u_{i}(s, x)-u_{i}^{*}(x)\right|^{p} d s d x .
\end{aligned}
$$

Let $\mathbb{R}$ be the weak infinitesimal operator; then for any given mode $r(t)=r \in S$, taking the derivative of $V_{1 i}(t, r)$ with respect to $t$ along the trajectory of (4) yields

$$
\begin{aligned}
& \mathcal{S} V_{1 i}(t, r) \\
& \quad=\lim _{\delta \rightarrow 0^{+}} \frac{1}{\delta}\left\{\mathbb { E } \left[\int_{\Omega} e^{\beta(t+\delta)} h_{i}(r(t+\delta))\left|u_{i}(t+\delta, x)-u_{i}^{*}\right|^{p} d x \mid\right.\right. \\
& \left.r(t)=r]-e^{\beta t} \int_{\Omega} h_{i}(r(t))\left|u_{i}(t, x)-u_{i}^{*}(x)\right|^{p} d x\right\} \\
& \quad=e^{\beta t}\left\{\int_{\Omega} h_{i}(r(t)) p\left|u_{i}-u_{i}^{*}\right|^{p-2}\left(u_{i}-u_{i}^{*}\right)\right. \\
& . \\
& \quad\left[\mathscr{D}_{i} \sum_{k=1}^{m}\left(\frac{\partial}{\partial x_{k}}\left(\left|\nabla u_{i}\right|^{p-2} \frac{\partial u_{i}}{\partial x_{k}}\right)-\frac{\partial}{\partial x_{k}}\left(\left|\nabla u_{i}^{*}\right|^{p-2} \frac{\partial u_{i}^{*}}{\partial x_{k}}\right)\right)\right. \\
& -\left(b_{i}\left(u_{i}\right)-b_{i}\left(u_{i}^{*}\right)\right)+\sum_{j=1}^{J} \rho_{j}(\omega(t)) c_{i j}^{r}\left(f_{i}\left(u_{i}\right)-f_{i}\left(u_{i}^{*}\right)\right) \\
& \left.+\sum_{j=1}^{J} \rho_{j}(\omega(t)) d_{i j}^{r}\left(g_{i}\left(u_{i}\left(t-\tau_{i}(t), x\right)\right)-g_{i}\left(u_{i}^{*}\right)\right)\right] d x \\
& \left.+\int_{\Omega} \sum_{k \in S} \pi_{i k} h_{i}(k)\left|u_{i}(t, x)-u_{i}^{*}\right|^{p} d x+\beta h_{i}(r(t))\right]_{\Omega} \mid u_{i} \\
& \left.+\left.u_{i}^{*}\right|^{p} d x\right\} .
\end{aligned}
$$

Next, we claim that

$$
\begin{gathered}
\sum_{k=1}^{m} \int_{\Omega} \mathscr{D}_{i}\left|u_{i}-u_{i}^{*}\right|^{p-2}\left(u_{i}-u_{i}^{*}\right)\left(\frac{\partial}{\partial x_{k}}\left(\left|\nabla u_{i}\right|^{p-2} \frac{\partial u_{i}}{\partial x_{k}}\right)\right. \\
\left.-\frac{\partial}{\partial x_{k}}\left(\left|\nabla u_{i}^{*}\right|^{p-2} \frac{\partial u_{i}^{*}}{\partial x_{k}}\right)\right) d x \leqslant 0 .
\end{gathered}
$$

To verify (40), we have to prove firstly the following proposition by the Yang inequality.

Proposition 7. For $\eta, \psi \in W_{0}^{1, p}(\Omega)$, one has

$$
\left(|\nabla \eta|^{p-2} \nabla \eta-|\nabla \psi|^{p-2} \nabla \psi\right)(\nabla \eta-\nabla \psi) \geqslant 0 .
$$


Proof. In fact, the Yang inequality yields

$$
\begin{aligned}
|\nabla \eta|^{p-2} \nabla \eta \nabla \psi & \leqslant|\nabla \eta|^{p-1}|\nabla \psi| \\
& \leqslant \frac{p-1}{p}\left(|\nabla \eta|^{p-1}\right)^{p /(p-1)}+\frac{|\nabla \psi|^{p}}{p}, \\
|\nabla \psi|^{p-2} \nabla \psi \nabla \eta & \leqslant \frac{p-1}{p}\left(|\nabla \psi|^{p-1}\right)^{p /(p-1)}+\frac{|\nabla \eta|^{p}}{p} .
\end{aligned}
$$

Synthesizing the above two inequalities results in (41).

So we can get by Gauss formula, the Dirichlet zeroboundary value, and Proposition 7

$$
\begin{aligned}
& \sum_{k=1}^{m} \int_{\Omega} \mathscr{D}_{i}\left|u_{i}-u_{i}^{*}\right|^{p-2}\left(u_{i}-u_{i}^{*}\right) \\
& \cdot\left(\frac{\partial}{\partial x_{k}}\left(\left|\nabla u_{i}\right|^{p-2} \frac{\partial u_{i}}{\partial x_{k}}\right)-\frac{\partial}{\partial x_{k}}\left(\left|\nabla u_{i}^{*}\right|^{p-2} \frac{\partial u_{i}^{*}}{\partial x_{k}}\right)\right) d x \\
& =-\sum_{k=1}^{m} \int_{\Omega} \mathscr{D}_{i}\left(\left|\nabla u_{i}\right|^{p-2} \frac{\partial u_{i}}{\partial x_{k}}-\left|\nabla u_{i}^{*}\right|^{p-2} \frac{\partial u_{i}^{*}}{\partial x_{k}}\right) \\
& \cdot \frac{\partial}{\partial x_{k}}\left(\left|u_{i}-u_{i}^{*}\right|^{p-2}\left(u_{i}-u_{i}^{*}\right)\right) d x=-\int_{\Omega} \mathscr{D}_{i}(p \\
& -1)\left|u_{i}-u_{i}^{*}\right|^{p-2}\left[\left(\left|\nabla u_{i}\right|^{p-2} \nabla u_{i}-\left|\nabla u_{i}^{*}\right|^{p-2} \nabla u_{i}^{*}\right)\right. \\
& \left.\cdot\left(\nabla u_{i}-\nabla u_{i}^{*}\right)\right] d x \leqslant 0,
\end{aligned}
$$

which proves (40).

In addition, we get by (A1)

$$
\begin{gathered}
\int_{\Omega} h_{i}(r(t)) p\left|u_{i}-u_{i}^{*}\right|^{p-2}\left(u_{i}-u_{i}^{*}\right) \\
\cdot\left(b_{i}\left(u_{i}\right)-b_{i}\left(u_{i}^{*}\right)\right) d x \geqslant h_{i}(r(t)) \\
\cdot p B_{i} \int_{\Omega}\left|u_{\mathrm{i}}-u_{i}^{*}\right|^{p} d x .
\end{gathered}
$$

Further, we can derive by (A2)

$$
\begin{aligned}
& \int_{\Omega} h_{i}(r(t)) p\left|u_{i}-u_{i}^{*}\right|^{p-2}\left(u_{i}-u_{i}^{*}\right) \\
& \cdot \sum_{j=1}^{J} \rho_{j}(\omega(t)) c_{i j}^{r}\left(f_{i}\left(u_{i}\right)-f_{i}\left(u_{i}^{*}\right)\right) d x \leqslant h_{i}(r(t)) \\
& \quad \cdot p F_{i}\left(\sum_{j=1}^{J}\left|c_{i j}^{r}\right|\right) \int_{\Omega}\left|u_{i}-u_{i}^{*}\right|^{p} d x .
\end{aligned}
$$

In addition, (A2) and the Yang inequality yield

$$
\begin{aligned}
& \int_{\Omega} h_{i}(r(t)) p\left|u_{i}-u_{i}^{*}\right|^{p-2}\left(u_{i}-u_{i}^{*}\right) \\
& . \sum_{j=1}^{J} \rho_{j}(\omega(t)) d_{i j}^{r}\left(g_{i}\left(u_{i}\left(t-\tau_{i}(t), x\right)\right)-g_{i}\left(u_{i}^{*}\right)\right) \\
& \leqslant h_{i}(r(t)) G_{i}\left(\sum_{j=1}^{J}\left|d_{i j}^{r}\right|\right)\left[(p-1) \int_{\Omega}\left|u_{i}-u_{i}^{*}\right|^{p} d x\right. \\
& \left.\quad+\int_{\Omega}\left|u_{i}\left(t-\tau_{i}(t), x\right)-u_{i}^{*}\right|^{p} d x\right] .
\end{aligned}
$$

Combining the above analyses results in

$$
\begin{aligned}
& \mathcal{L} V_{1 i}(t, r) \leqslant e^{\beta t}\left[-a_{i}(r) \int_{\Omega}\left|u_{i}-u_{i}^{*}\right|^{p} d x\right. \\
& \left.\quad+b_{i}(r) \int_{\Omega}\left|u_{i}\left(t-\tau_{i}(t), x\right)-u_{i}^{*}\right|^{p} d x\right], \quad \forall r \in S .
\end{aligned}
$$

On the other hand, we have

$$
\begin{aligned}
\mathfrak{Q} V_{2 i} \leqslant & \frac{b_{i}}{1-K} e^{\beta \tau} \int_{\Omega} e^{\beta t}\left|u_{i}(t, x)-u_{i}^{*}(x)\right|^{p} d x \\
& -b_{i} \int_{\Omega} e^{\beta t}\left|u_{i}\left(t-\tau_{i}(t), x\right)-u_{i}^{*}(x)\right|^{p} d x .
\end{aligned}
$$

Thus, we get by (14)

$$
\begin{aligned}
\mathfrak{L} V_{i}(t, r(t)) & \leqslant e^{\beta t}\left(-a_{i}+\frac{b_{i}}{1-K} e^{\beta \tau}\right) \int_{\Omega}\left|u_{i}-u_{i}^{*}\right|^{p} d x \\
& \leqslant 0, \quad \forall t \geqslant 0 .
\end{aligned}
$$

So we can obtain by the Dynkin formula

$$
\begin{aligned}
& E V(t, r(t))-E V(0, r(0))=E \int_{0}^{t} \mathfrak{Q} V(s, r(s)) d s \\
& \quad \leqslant 0, \quad t \geqslant 0 .
\end{aligned}
$$

Hence, we have

$$
\begin{aligned}
& \left(\min _{r \in S} h_{i}(r(t))\right) E\left(e^{\beta t} \int_{\Omega}\left|u_{i}(t, x)-u_{i}^{*}(x)\right|^{p} d x\right) \\
& \quad \leqslant E V(t, r(t)) \leqslant E V(0, r(0)) \\
& \quad \leqslant E\left(\max _{r \in S} h_{i}(r(t)) \int_{\Omega}\left|u_{i}(0, x)-u_{i}^{*}(x)\right|^{p} d x\right. \\
& \left.\quad+\frac{b_{i}}{1-K} e^{\beta \tau} \int_{\Omega} \int_{-\tau}^{0} e^{\beta s}\left|u_{i}(s, x)-u_{i}^{*}(x)\right|^{p} d s d x\right) \\
& \quad \leqslant\left(\max _{r \in S} h_{i}(r(t))+\frac{b_{i}}{1-K} e^{\beta \tau}\right) \\
& \cdot \sup _{-\tau \leqslant s \leqslant 0} E\left\|\phi_{i}(s)-u_{i}^{*}\right\|_{L^{p}}^{p},
\end{aligned}
$$


which implies

$$
\begin{aligned}
& E\left(\int_{\Omega}\left|u_{i}(t, x)-u_{i}^{*}(x)\right|^{p} d x\right) \\
& \quad \leqslant h_{i} e^{-\beta t} \sup _{-\tau \leqslant s \leqslant 0} E\left\|\phi_{i}(s)-u_{i}^{*}\right\|_{L^{p}}^{p}, \quad i \in \mathcal{N},
\end{aligned}
$$

where $h_{i}=\left(\max _{r \in S} h_{i}(r(t))+\left(b_{i} /(1-K)\right) e^{\beta \tau}\right) / \min _{r \in S} h_{i}(r(t))>$ $0, \forall i \in \mathcal{N}$. Then we have

$$
\begin{aligned}
E\left\|u-u^{*}\right\|_{L^{p}}^{p} & =\sum_{i \in \mathcal{N}} E\left(\int_{\Omega}\left|u_{i}(t, x)-u_{i}^{*}(x)\right|^{p} d x\right) \\
& \leqslant\left(\max _{i \in \mathcal{N}} h_{i}\right) e^{-\beta t} E\left\|\phi-u^{*}\right\|_{\tau}^{p},
\end{aligned}
$$

where we denote $E\left\|\phi-u^{*}\right\|_{\tau}^{p}=\sum_{i \in \mathcal{N}} \sup _{-\tau \leqslant s \leqslant 0}\left(E \| \phi_{i}(s)-\right.$ $\left.u_{i}^{*} \|_{L^{p}}^{p}\right)$.

Now, we can conclude from Definition 2 that the nonconstant equilibrium solution of (4) is stochastically exponentially $p$-stable about $L^{p}$ norm. And that completes the proof of Theorem 6.

Remark 8. In [27], existence theorems of stochastic differential equations on $t \in\left[t_{0}, T\right)$ were given under some conditions on activation functions, where $T>0$ is a constant. And in $[28,29]$, existence theorems of stochastic differential equations were presented under some conditions on function $V \in C^{1,2}\left(\left[t_{0}-\tau, T\right) \times R^{n} ; R_{+}\right)$. Motivated by [27], we proposed some conditions on activation functions to set up existence criterion for the equilibrium solution of system (4). In [22, 23], the constant equilibrium solution $u=u^{*}$ for all $t \in$ $\left[t_{0},+\infty\right)$ was obtained by homomorphic mapping theory and matrix theory, or matrix theory and homotopy invariance theorem, where $u^{*}=\left(u_{1}^{*}, u_{2}^{*}, \ldots, u_{i}^{*}, \ldots, u_{n}^{*}\right)$, and each $u_{i}^{*}$ is a constant. In this paper, we also need to consider the equilibrium solution of (4) defined on $\left[t_{0},+\infty\right)$. Different from $[22,23]$, we consider the nonconstant equilibrium solution $u=u^{*}(x)=\left(u_{1}^{*}(x), u_{2}^{*}(x), \ldots, u_{i}^{*}(x), \ldots, u_{n}^{*}(x)\right)$ for all $t \in\left[t_{0},+\infty\right)$. This equilibrium solution is a solution for a nonlinear $p$-Laplacian elliptic partial differential equation whose space frame may be considered as infinite dimension function space $W_{0}^{1, p}(\Omega)$. And variational method is always a powerful tool to solve the problem. Although the variational method is more complicated than homomorphic mapping method, $M$-matrix method, or homotopy invariance theorem, our criterion about existence is only involved in the activation functions (remark: condition (14) is not used in the proof of existence) and hence is simpler and more effective than other criteria, such as $M$-matrix criteria and LMI-based criteria, because LMI-based criteria or $M$-matrix criteria always involve the computer MATLAB programming in practical application while our condition (13) is easy to verify. So our existence criterion is actually simpler and more effective than LMI-based criteria and other criteria, which is the main contribution in this paper.

Remark 9. LMI-based stability criteria or $M$-matrix stability criteria are always proposed in many literatures related to the mean square stability (see, e.g., [30-33] and references therein). However, when $p>1$ and $p \neq 2, p$-stability criteria always involve more complicated mathematical method and mathematical deduction. For example, the stability criteria in [34] are not simpler than our stability criterion in Theorem 6. Similar phenomena exposed in many literatures related to $p$ stability (see $[15,34-38]$ ). Besides, the nonlinear $p$-Laplacian $(p>1)$ operator produces great difficulties in $p$-stability proof. However, our condition (14) is still a LMI condition, which can be computed and verified by computer MATLAB LMI Toolbox in practical application.

\section{Numerical Example}

Example 1. Consider the 5-Laplace fuzzy T-S dynamical equations as follows.

Fuzzy Ruler 1 . IF $\omega_{1}(t)$ is $1 / e^{-2 \omega_{1}(t)}$, THEN

$$
\begin{aligned}
& \frac{d u_{1}(t, x)}{d t} \\
& =\mathscr{D}_{1} \operatorname{div}\left(\left|\nabla u_{1}\right|^{3} \nabla u_{1}\right)-b_{1}\left(u_{1}\right) \\
& \quad+\left(c_{11}^{r} f_{1}\left(u_{1}(t, x)\right)+d_{11}^{r} g_{1}\left(u_{1}\left(t-\tau_{1}(t), x\right)\right)\right) \\
& \quad+I_{1}, \quad t>0, x \in \Omega, \\
& \frac{d u_{2}(t, x)}{d t} \\
& \quad+\left(c_{21}^{r} f_{2}\left(u_{2}(t, x)\right)+d_{21}^{r} g_{2}\left(u_{2}\left(t-\tau_{2}(t), x\right)\right)\right) \\
& \quad+I_{2}, \quad t>0, x \in \Omega, \\
& u_{i}(t, x)=0, \quad i \in \mathcal{N}, t \geqslant 0, x \in \partial \Omega .
\end{aligned}
$$

Fuzzy Ruler 2. IF $\omega_{2}(t)$ is $1-1 / e^{-2 \omega_{1}(t)}$, THEN

$$
\begin{aligned}
& \frac{d u_{1}(t, x)}{d t} \\
& =\mathscr{D}_{1} \operatorname{div}\left(\left|\nabla u_{1}\right|^{3} \nabla u_{1}\right)-b_{1}\left(u_{1}\right) \\
& \quad+\left(c_{12}^{r} f_{1}\left(u_{1}(t, x)\right)+d_{12}^{r} g_{1}\left(u_{1}\left(t-\tau_{1}(t), x\right)\right)\right) \\
& \quad+I_{1}, \quad t>0, x \in \Omega, \\
& \frac{d u_{2}(t, x)}{d t} \\
& \quad+\left(c_{22}^{r} f_{2}\left(u_{2}(t, x)\right)+d_{22}^{r} g_{2}\left(u_{2}\left(t-\tau_{2}(t), x\right)\right)\right) \\
& \quad+I_{2}, \quad t>0, x \in \Omega, \\
& u_{i}(t, x)=0, \quad i \in \mathcal{N}, t \geqslant 0, \quad x \in \partial \Omega
\end{aligned}
$$



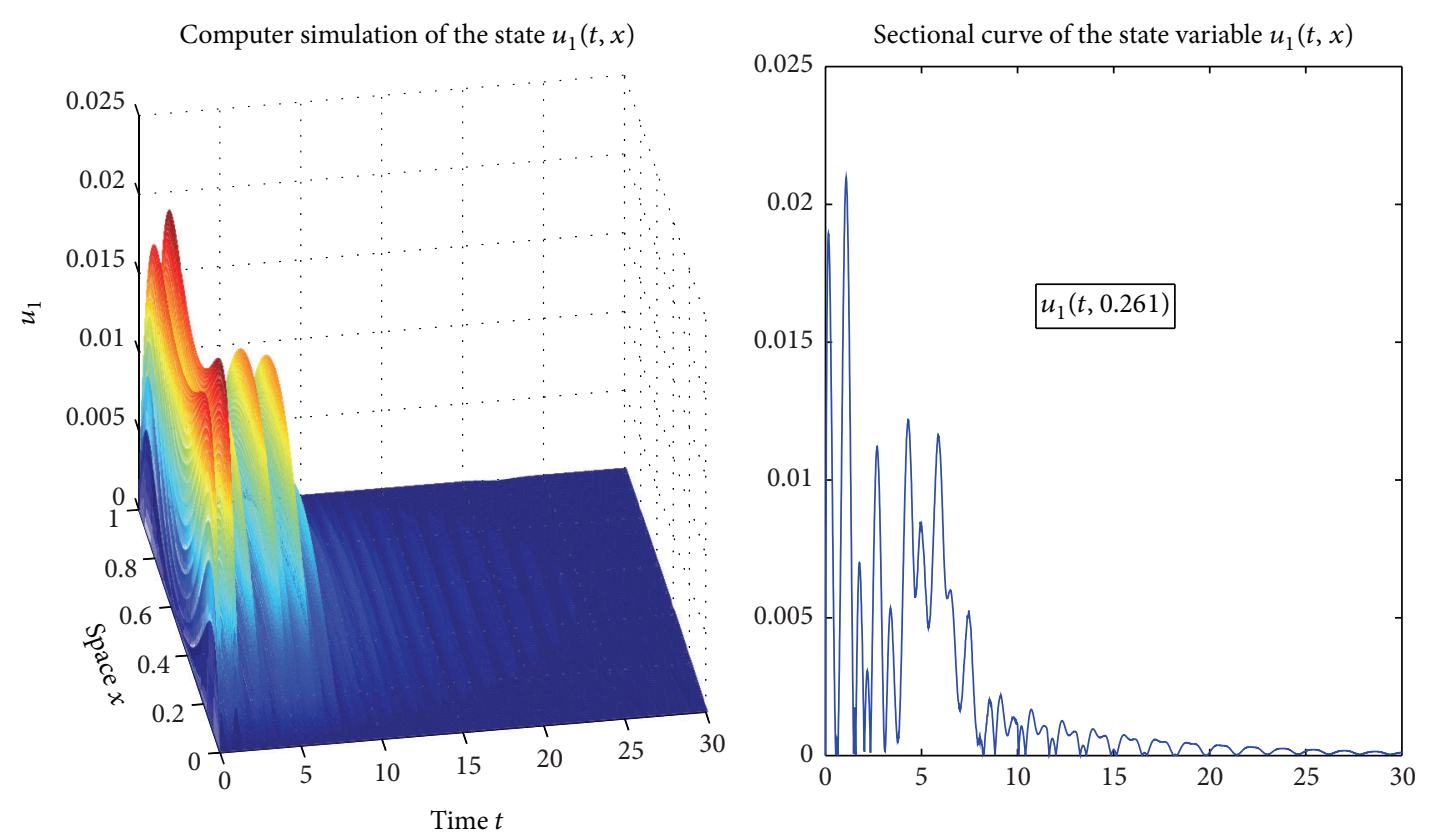

Figure 1: The state variable $u_{1}(t, x)$.

equipped with the initial value

$$
\phi(s, x)=\left(\begin{array}{c}
0.2 \cos (2 \pi x(1-x))^{2} \sin (\pi s)^{2} \\
0.25 \sin (6 x(1-x))^{2} \cos (3 \pi s)^{2}
\end{array}\right),
$$

$-83 \leq s \leq 0$,

where $x \in \Omega \triangleq\left\{\left(x_{1}, x_{2}\right)^{T} \in R^{2}:\left|x_{i}\right|<1, i \in \mathcal{N}\right\}, N=\{1,2\}$, $r \in S \triangleq\{1,2\}, c_{11}^{1}=c_{21}^{1}=0.1, c_{11}^{2}=c_{21}^{2}=0.11, c_{12}^{1}=c_{22}^{1}=$ $0.15, c_{12}^{2}=0.12=c_{22}^{2}, d_{11}^{1}=d_{21}^{1}=0.1, d_{11}^{2}=d_{21}^{2}=0.11$, $d_{12}^{1}=d_{22}^{1}=0.15, d_{12}^{2}=0.12=d_{22}^{2}, b_{1}\left(u_{1}\right)=u_{1}^{3}+(1 / 2) u_{1}$, $b_{2}\left(u_{2}\right)=u_{2}^{3}+(1 / 3) u_{2}$, and $B_{1}=1 / 2$, and $B_{2}=1 / 3$. Consider $f_{1}\left(u_{1}\right)=0.2 u_{1} \sin u_{1}=g_{1}\left(u_{1}\right), f_{2}\left(u_{2}\right)=0.3 u_{2}+0.2 \cos u_{2}=$ $g_{2}\left(u_{2}\right)$, and $F_{1}=G_{1}=0.4, F_{2}=G_{2}=0.5$; let $\tau_{i}(t) \equiv 83, i \in$ $\mathcal{N}$, and $\tau=83, K=0$; denote $h_{i}(r)=h_{i}^{r}, r \in S$. Let $h_{1}^{1}=$ $1, h_{2}^{1}=2, h_{1}^{2}=3, h_{2}^{2}=4$, and $\beta=0.01$. Denote $a_{i}(r)=a_{i}^{r}$ and $b_{i}(r)=b_{i}^{r}$; then we can compute by computer MATLAB that $a_{1}^{1}=1.5900, a_{2}^{1}=4.9860, a_{1}^{2}=1.0633, a_{2}^{2}=2.4867$, $b_{1}^{1}=0.1000, b_{2}^{1}=0.2760, b_{1}^{2}=0.2500$, and $b_{2}^{2}=0.4600$, and hence $a_{1}=1.5900, a_{2}=1.0633, b_{1}=0.2760, b_{2}=0.4600$, and

$$
\begin{aligned}
& -a_{1}+\frac{b_{1}}{1-K} e^{\beta \tau}=-0.9570<0, \\
& -a_{2}+\frac{b_{2}}{1-K} e^{\beta \tau}=-0.0084<0,
\end{aligned}
$$

which imply that condition (14) is satisfied. In addition, condition (13) is obviously satisfied. Therefore, there exists a nonconstant equilibrium solution for PDEs (54a)-(54b), which is stochastically global exponential $p$-stability about $L^{p}$ norm (see Figures 1 and 2).

\section{Conclusions}

The nonlinear $p$-Laplace $(p>1, p \neq 2)$ brings great difficulties to the proof of the existence of the $p$-stability nonconstant equilibrium solution, for $p$-Laplace $(p>1, p \neq 2)$ partial differential equations (PDEs) always need be considered in Banach space $W^{1, p}(\Omega)$ while the common linear Laplace $(p=$ 2) PDEs can be studied in the setting of the special Hilbert space $H^{1}$ that can be orthogonally decomposed into the direct sum of the eigenfunction spaces. However, by applying Ekeland variational principle and the Yang inequality and constructing the suitable Lyapunov functional, we overcome those difficulties to obtain the existence of exponential $p$ stability nonconstant equilibrium solution for Markovian jumping $p$-Laplace $(p>1)$ partial differential equations (PDEs) (4) under Dirichlet boundary condition. As far as we know, it is the first time to apply the Ekeland variational principle to solve the above problem so that some methods used in this paper are different from those of many previous related literatures (see Remark 1). In addition, the obtained existence criterion is only involved in the activation functions so that the criterion is simpler and easier than other existence criteria to be verified in practical application. Moreover, a numerical example shows the effectiveness of the proposed methods owing to the large allowable variation range of timedelay.

\section{Conflict of Interests}

The authors declare that there is no conflict of interests regarding the publication of this paper.

\section{Acknowledgments}

The authors are very thankful to the anonymous reviewers for their suggestions, which improved the quality of this paper. 

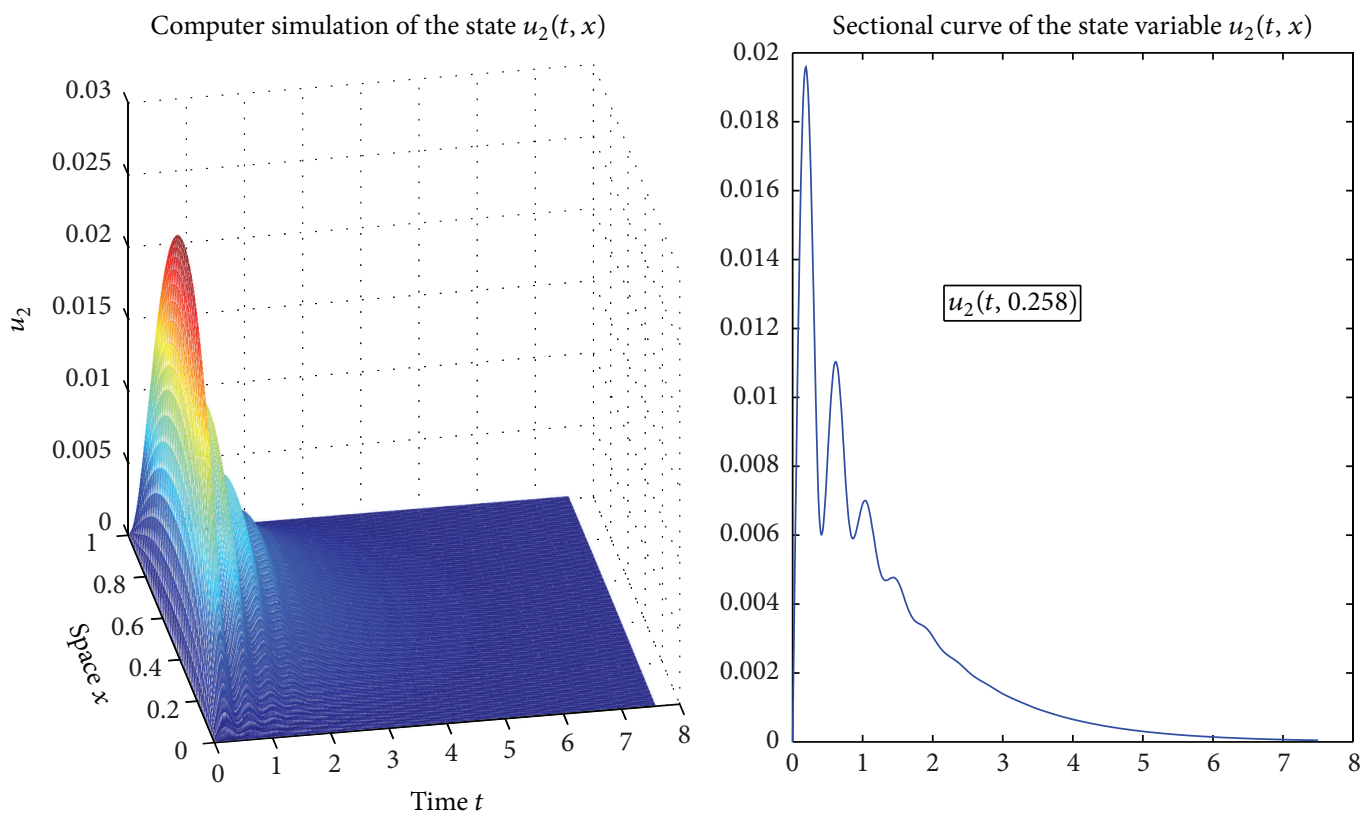

FIGURE 2: The state variable $u_{2}(t, x)$.

This work was supported by the National Basic Research Program of China (2010CB732501), by the Scientific Research Fund of Science Technology Department of Sichuan Province (2012JY010), and by Sichuan Educational Committee Science Foundation (08ZB002, 12ZB349, and 14ZA0274).

\section{References}

[1] Y. H. Li, Y. S. Mi, and C. Mu, "Properties of positive solutions for a nonlocal non-linear diffusion equation with nonlocal nonlinear boundary condition," Acta Mathematica Scientia, vol. 34, no. 3, pp. 748-758, 2014.

[2] Y. Wang, C. Mu, and Z. Xiang, "Blowup of solutions to a porous medium equation with nonlocal boundary condition," Applied Mathematics and Computation, vol. 192, no. 2, pp. 579-585, 2007.

[3] V. A. Galaktionov, "On asymptotic self-similar behaviour for a quasilinear heat equation: single point blow-up," SIAM Journal on Mathematical Analysis, vol. 26, no. 3, pp. 675-693, 1995.

[4] A. A. Samarskii, S. P. Kurdyumov, V. A. Galaktionov, and A. P. Mikhailov, Blow-Up in Problems for Quasilinear Parabolic Equations, Nauka, Moscow, Russia, 1987, Walter de Gruyter, Berlin, Germany, 1995.

[5] R. S. Cantrell and C. Cosner, "Diffusive logistic equations with indefinite weights: population models in disrupted environments II," SIAM Journal on Mathematical Analysis, vol. 22, no. 4, pp. 1043-1064, 1989.

[6] Z. Q. Wu, J. N. Zhao, J. X. Yin, and H. L. Li, Nonlinear Diffusion Equations, World Scientific Publishing, River Edge, NJ, USA, 2001.

[7] P. Qingfei, Z. Zifang, and H. Jingchang, "Stability of the stochastic reaction-diffusion neural network with time-varying delays and P-laplacian," Journal of Applied Mathematics, vol. 2012, Article ID 405939, 10 pages, 2012.

[8] R. Rao, S. Zhong, and X. Wang, "Stochastic stability criteria with LMI conditions for Markovian jumping impulsive BAM neural networks with mode-dependent time-varying delays and nonlinear reaction-diffusion," Communications in Nonlinear Science and Numerical Simulation, vol. 19, no. 1, pp. 258-273, 2014.

[9] X. R. Wang, R. F. Rao, and S. M. Zhong, "LMI approach to stability analysis of Cohen-Grossberg neural networks with $p$ Laplace diffusion," Journal of Applied Mathematics, vol. 2012, Article ID 523812, 12 pages, 2012.

[10] R. Sathy and P. Balasubramaniam, "Stability analysis of fuzzy Markovian jumping Cohen-Grossberg BAM neural networks with mixed time-varying delays," Communications in Nonlinear Science and Numerical Simulation, vol. 16, no. 4, pp. 2054-2064, 2011.

[11] H. Y. Liu, Y. Ou, J. Hu, and T. Liu, "Delay-dependent stability analysis for continuous-time BAM neural networks with Markovian jumping parameters," Neural Networks, vol. 23, no. 3, pp. 315-321, 2010.

[12] X. Liu and H. Xi, "Stability analysis for neutral delay Markovian jump systems with nonlinear perturbations and partially unknown transition rates," Advances in Mathematical Physics, vol. 2013, Article ID 592483, 20 pages, 2013.

[13] T. Takagi and M. Sugeno, "Fuzzy identification of systems and its applications to modeling and control," IEEE Transactions on Systems, Man and Cybernetics, vol. 15, no. 1, pp. 116-132, 1985.

[14] K. Tanaka and M. Sugeno, "Stability analysis and design of fuzzy control systems," Fuzzy Sets and Systems, vol. 45, no. 2, pp. 135156, 1992.

[15] S. Long and D. Xu, "Global exponential p-stability of stochastic non-autonomous Takagi-Sugeno fuzzy cellular neural networks with time-varying delays and impulses," Fuzzy Sets and Systems, vol. 253, pp. 82-100, 2014.

[16] Z. Chen and D. Zhao, "Stabilization effect of diffusion in delayed neural networks systems with Dirichlet boundary conditions," Journal of the Franklin Institute, vol. 348, no. 10, pp. 2884-2897, 2011. 
[17] L. Wang and D. Xu, "Global exponential stability of Hopfield reaction-diffusion neural networks with time-varying delays," Science in China Series F: Information Sciences, vol. 46, no. 6, pp. 466-474, 2003.

[18] Y. G. Kao, C. H. Wang, H. R. Karimi, and R. Bi, "Global stability of coupled Markovian switching reaction-diffusion systems on networks," Nonlinear Analysis: Hybrid Systems, vol. 13, pp. 61-73, 2014.

[19] C. Xu and J. J. Wei, "On stability of two degenerate reactiondiffusion systems," Journal of Mathematical Analysis and Applications, vol. 390, no. 1, pp. 126-135, 2012.

[20] C. H. Zhou, H. Y. Zhang, H. B. Zhang, and C. Y. Dang, "Global exponential stability of impulsive fuzzy Cohen-Grossberg neural networks with mixed delays and reaction-diffusion terms," Neurocomputing, vol. 91, pp. 67-76, 2012.

[21] P. Lindqvise, "On the equation $\operatorname{div}\left(|\nabla u|^{p-2}\right)+\lambda|u|^{p-2} u=0$," Proceedings of the American Mathematical Society, vol. 109, pp. 159-164, 1990.

[22] H. Chen, S. M. Zhong, and J. L. Shao, "Exponential stability criterion for interval neural networks with discrete and distributed delays," Applied Mathematics and Computation, vol. 250, pp. 121-130, 2015.

[23] X. H. Zhang and K. L. Li, "Integro-differential inequality and stability of BAM FCNNs with time delays in the leakage terms and distributed delays," Journal of Inequalities and Applications, vol. 2011, article 43, 2011.

[24] I. Ekeland, "Nonconvex minimization problems," Bulletin of the American Mathematical Society, vol. 1, no. 3, pp. 443-474, 1979.

[25] P. Drabek, Solvability and Bifurcations of Nonlinear Equations, vol. 265 of Pitman Research Notes in Mathematics Series, Longman Harlow, 1992.

[26] P. Drabek, A. Kufner, and F. Nicolosi, Quasilinear Elliptic Equations with Degenerations and Singularities, Walter De Gruyter, Berlin, Germany, 1997.

[27] D. Y. Xu, X. H. Wang, and Z. G. Yang, "Further results on existence-uniqueness for stochastic functional differential equations," Science China Mathematics, vol. 56, no. 6, pp. 11691180, 2013.

[28] D. Y. Xu, B. Li, S. J. Long, and L. Y. Teng, "Moment estimate and existence for solutions of stochastic functional differential equations," Nonlinear Analysis: Theory, Methods \& Applications, vol. 108, pp. 128-143, 2014.

[29] D. Y. Xu, B. Li, S. J. Long, and L. Y. Teng, "Corrigendum to 'Moment estimate and existence for solutions of stochastic functional differential equations' [Nonlinear Anal.: TMA 108 (2014) 128-143]," Nonlinear Analysis: Theory, Methods \& Applications, vol. 114, pp. 128-143, 2015.

[30] H. G. Zhang, Z. S. Wang, and D. R. Liu, "A comprehensive review of stability analysis of continuous-time recurrent neural networks," IEEE Transactions on Neural Networks and Learning Systems, vol. 25, no. 7, pp. 1229-1262, 2014.

[31] H. Zhang and Y. Wang, "Stability analysis of Markovian jumping stochastic Cohen-Grossberg neural networks with mixed time delays," IEEE Transactions on Neural Networks, vol. 19, no. 2, pp. 366-370, 2008.

[32] J. K. Tian, Y. M. Li, J. Z. Zhao, and S. M. Zhong, "Delaydependent stochastic stability criteria for Markovian jumping neural networks with mode-dependent time-varying delays and partially known transition rates," Applied Mathematics and Computation, vol. 218, no. 9, pp. 5769-5781, 2012.
[33] S. J. Long and D. Y. Xu, "Global exponential stability of nonautonomous cellular neural networks with impulses and timevarying delays," Communications in Nonlinear Science and Numerical Simulation, vol. 18, no. 6, pp. 1463-1472, 2013.

[34] B. Li and D. Y. Xu, "Exponential p-stability of stochastic recurrent neural networks with mixed delays and Markovian switching," Neurocomputing, vol. 103, pp. 239-246, 2013.

[35] L. Hu, Y. Ren, and T. Xu, "P-Moment stability of solutions to stochastic differential equations driven by $G$-Brownian motion," Applied Mathematics and Computation, vol. 230, pp. 231-237, 2014.

[36] X. H. Wang, Q. Y. Guo, and D. Y. Xu, "Exponential p-stability of impulsive stochastic Cohen-Grossberg neural networks with mixed delays," Mathematics and Computers in Simulation, vol. 79, no. 5, pp. 1698-1710, 2009.

[37] D. S. Li, X. H. Wang, and D. Y. Xu, "Existence and global pexponential stability of periodic solution for impulsive stochastic neural networks with delays," Nonlinear Analysis: Hybrid Systems, vol. 6, no. 3, pp. 847-858, 2012.

[38] Z. G. Yang, D. Y. Xu, and L. Xiang, "Exponential p-stability of impulsive stochastic differential equations with delays," Physics Letters A, vol. 359, no. 2, pp. 129-137, 2006. 


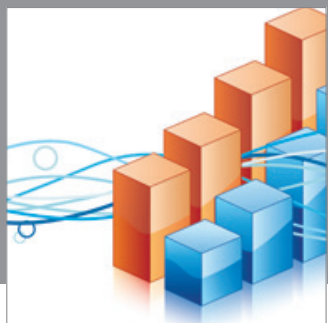

Advances in

Operations Research

mansans

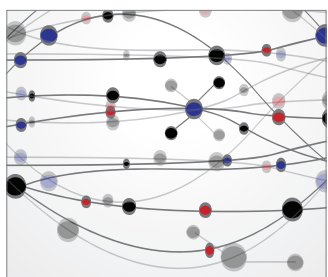

The Scientific World Journal
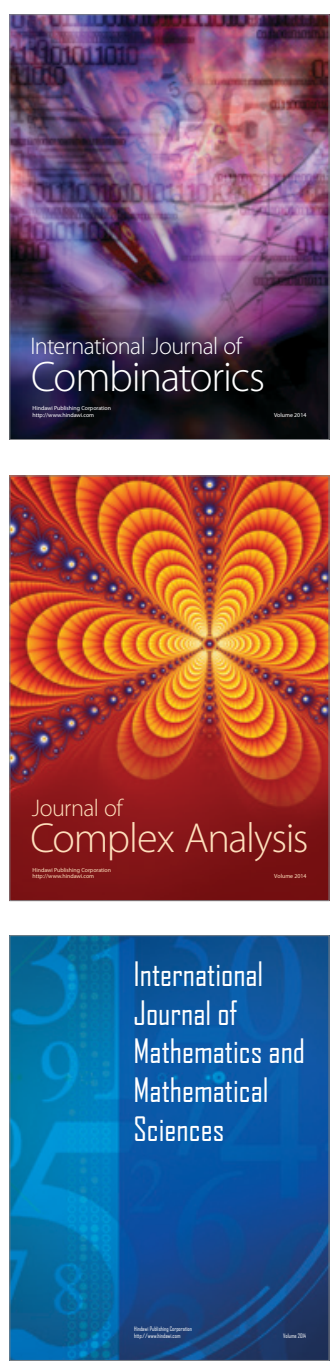
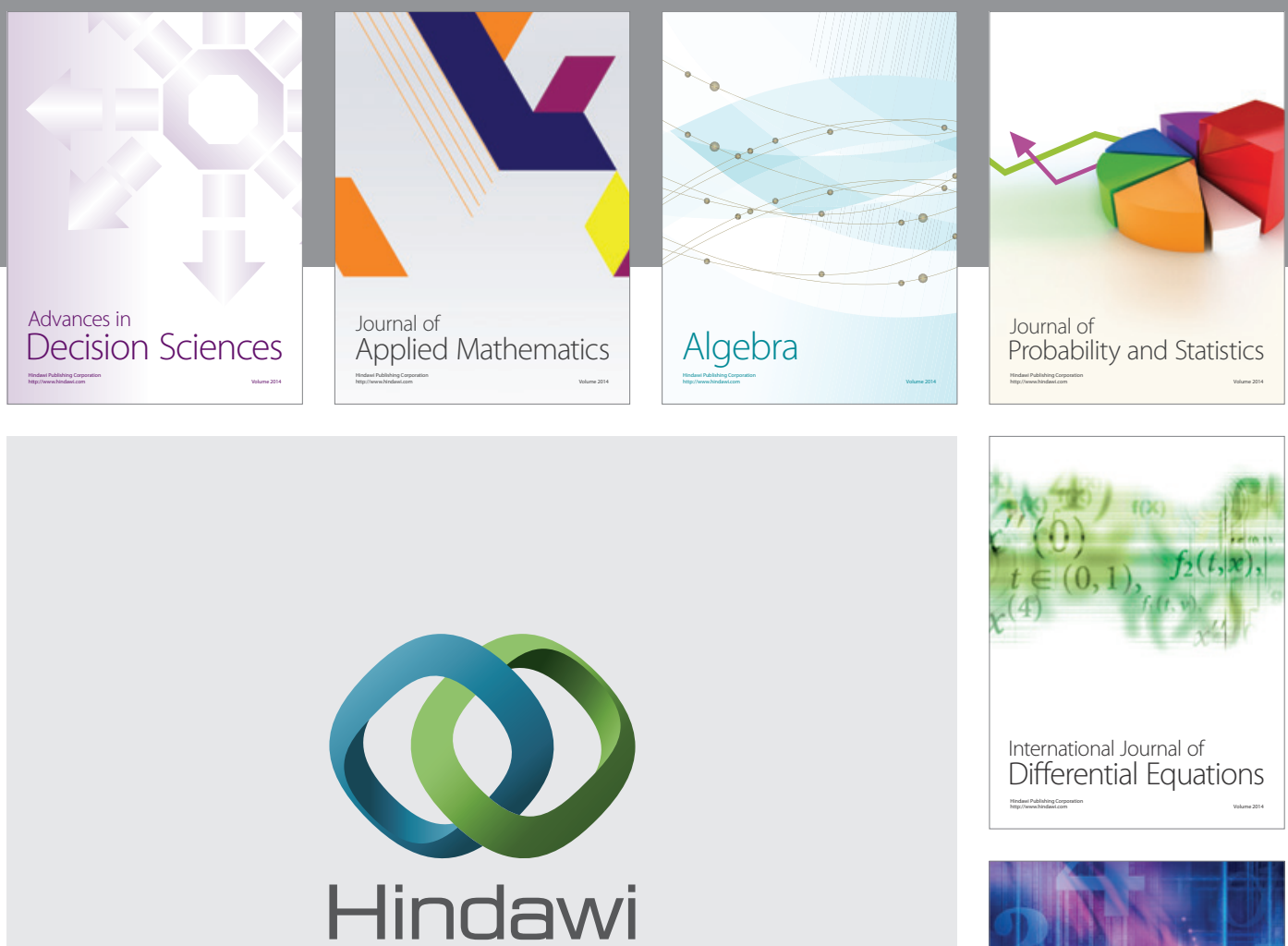

Submit your manuscripts at http://www.hindawi.com
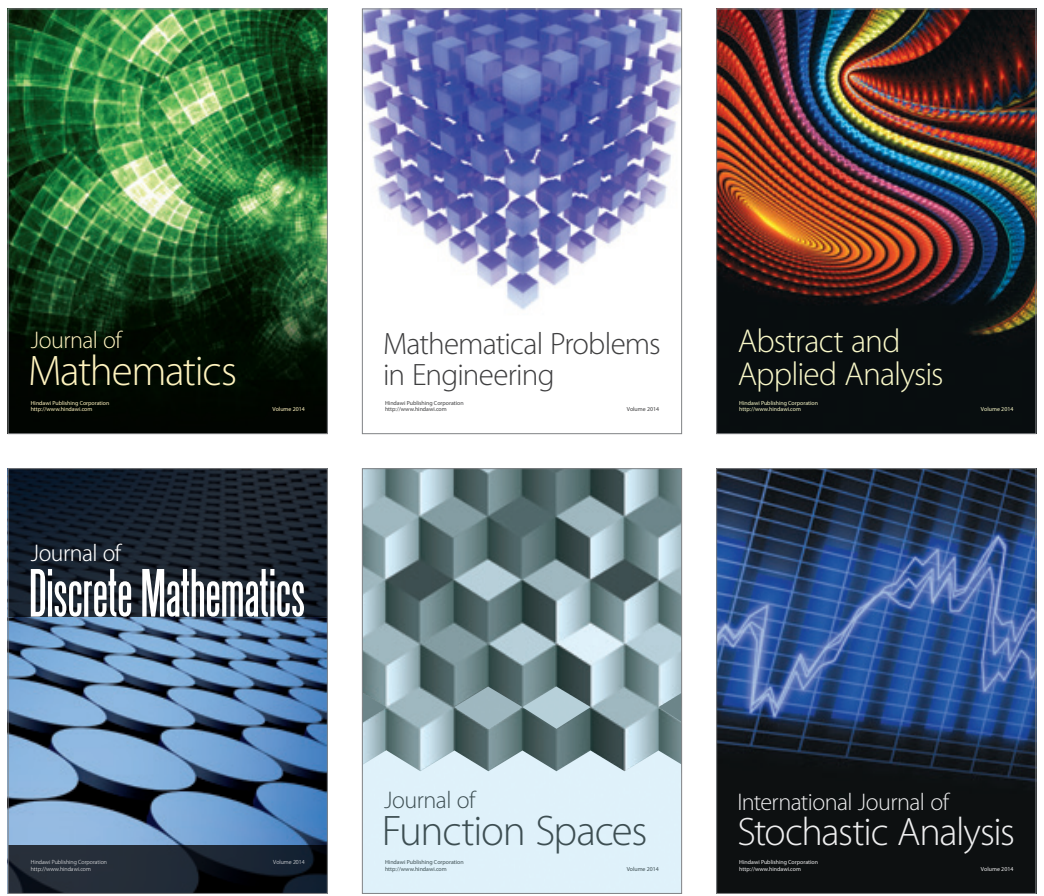

Journal of

Function Spaces

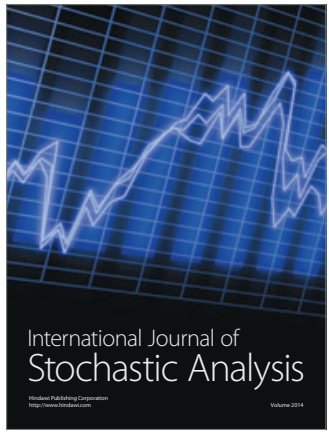

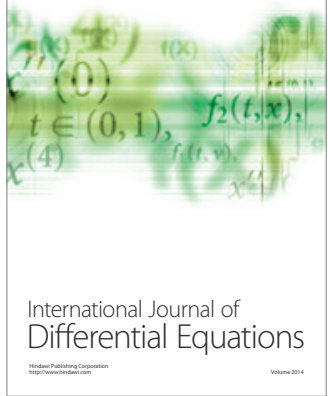
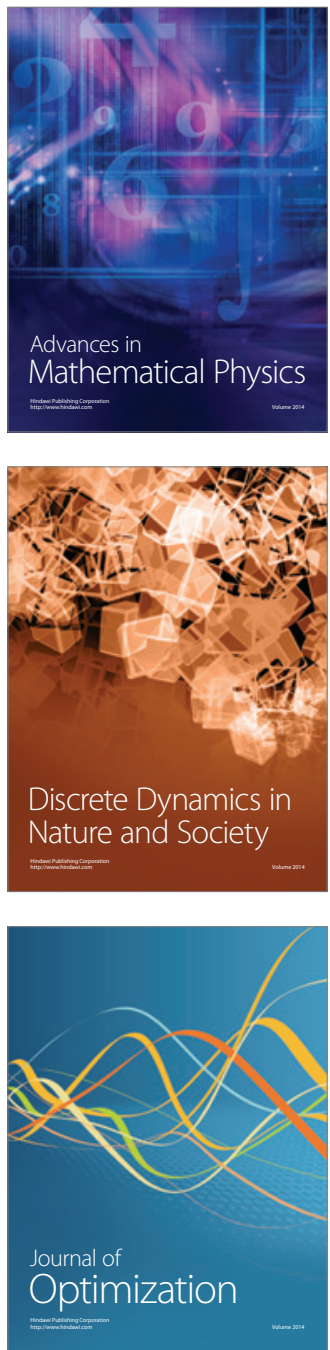